\title{
Going from A to B: the safety of incompatible group A plasma for emergency release in trauma and massive transfusion patients
}

$\mathrm{L}$

ike the Halloween nor'easter of 1991, AB plasma is currently facing a perfect storm of competing forces that is exacerbating the availability of an already limited resource. One force is AABB Standard 5.4.1.2, which becomes effective for all plasma and whole blood collected after April 1, 2014., ${ }^{1,2}$ Although TRALI reduction strategies have already been widely implemented for group A, B, and O donors for several years, they have generally not been applied to group $A B$ donors due to concerns of $\mathrm{AB}$ plasma availability. The riskbenefit of exempting $\mathrm{AB}$ donors was recently reassessed in the face of epidemiology studies examining the efficacy of TRALI reduction strategies. In general, TRALI cases and TRALI fatalities have decreased dramatically over the past several years for most products with the striking exception of $\mathrm{AB}$ plasma. ${ }^{2}$ In a recent 4 -year retrospective study of TRALI cases reported to the American Red Cross, the odds of TRALI with AB plasma were 14.5 -fold higher than that of plasma from other blood types (26.3 vs. 1.8 cases per million units). ${ }^{3}$ The application of Standard 5.4.1.2 to AB donors is projected to decrease $\mathrm{AB}$ plasma availability by $27 \%$ to $33 \%$, with $\mathrm{AB}$ donors making up only $2.6 \%$ of active donors. ${ }^{2}$

The second storm battering the AB plasma supply is the widespread adoption of massive transfusion protocols (MTPs), which advocate early and aggressive plasma resuscitation. In an informal poll of recent AABB annual meeting attendees, 93\% had MTPs with fixed plasma:RBC ratios. ${ }^{4}$ Moreover, $73 \%$ maintain a thawed plasma inventory with most hospitals providing only $\mathrm{AB}$ plasma to patients without a current or historical ABO type. Because many trauma patients require transfer to large regional trauma centers, these patients are more likely to initially receive $\mathrm{AB}$ plasma due to the absence of a historical $\mathrm{ABO}$ type on file at the receiving facility. ${ }^{5}$ The direct consequence is overutilization of $\mathrm{AB}$ plasma since most patients will be group A, O, or B. In addition, the use of fixed plasma:RBC ratios often leads to plasma overtransfusion. In one study, $86 \%$ of transfused trauma patients did not meet the definition of massive transfusion, averaging only three RBC transfusions in the first 12 hours. ${ }^{6}$ Finally, the need to maintain thawed $\mathrm{AB}$ plasma inventories increases $\mathrm{AB}$ utilization and transfusion to non- $\mathrm{AB}$ patients, as

(C) $2014 \mathrm{AABB}$

TRANSFUSION 2014;54:1695-1697. unused plasma is diverted to other patients to prevent wastage. $^{7}$ An analysis at Dartmouth determined that maintenance of a thawed, 2-unit $\mathrm{AB}$ plasma inventory would increase yearly $\mathrm{AB}$ utilization by at least $25 \% .{ }^{8}$ This estimate closely mirrors the overall increase in $\mathrm{AB}$ plasma use nationwide, which has risen $27 \%$ since $2006 .^{7}$

Several suggestions have been offered to minimize the impact of Standard 5.4.1.2 on AB plasma supplies. Among the most controversial proposals is the initial use of prethawed group A plasma for emergency release in trauma and other MTP patients. ${ }^{2,7}$ Proponents of the practice argue that most patients will be compatible with group A plasma, based on the known distribution of $A B O$ types in the population. Moreover, anti-B titers are relatively low in most group A donors, especially male-only donors who should lack immune-stimulated anti-B. ${ }^{9-11}$ Secretors may have additional protection from hemolysis due to the presence of $B$ substance capable of adsorbing anti-B from transfused plasma. ${ }^{11}$ As evidence, they point to the rarity of hemolytic transfusion reactions due to incompatible group A PLT transfusions. ${ }^{12}$ Finally, patients requiring an emergent MTP "pack" are also receiving several units of group O RBCs, which further decreases the risk of hemolysis.

In this issue, Chhibber and colleagues ${ }^{13}$ describe their 5-year experience using prethawed group A plasma for emergency release at the University of Massachusets. To monitor the safety of the policy, patients receiving incompatible plasma were prospectively monitored for clinical and laboratory signs of hemolysis. Between 2008 and 2013, a total of 358 patients required emergency release, group A plasma, representing less than $1 \%$ of their emergency room admissions (135,000 per year). As expected, most patients (84\%) were group A or group $\mathrm{O}$ and therefore $\mathrm{ABO}$ compatible with group A plasma. Group A plasma was dispensed to 54 group $\mathrm{B}$ and $\mathrm{AB}$ patients; however, only $23(6 \%)$ patients actually received group A-incompatible plasma. Most patients received less than 4 units of plasma including 10 patients who required plasma for warfarin reversal with little or no group O RBC support. Nearly half of patients $(11 / 23,48 \%)$ also received group O RBCs, with nine of 11 receiving at least 4 units. Only three patients, however, approached the definition of massive transfusion, receiving between 8 and 12 units of group O RBCs. There was no overt evidence of acute hemolytic transfusion reactions although three patients had weak positive posttransfusion DATs. 
Two other institutions have published their experience or analysis of prethawed group A plasma.,8 Both Mayo Clinic and Dartmouth serve as regional trauma centers for a geographically large rural population. In the first 3 years, Mayo dispensed group A plasma to 254 patients without an $\mathrm{ABO}$ type on file. ${ }^{5}$ Like the patients in the study by Chhibber and coworkers, most patients were group A or group O (86\%) and therefore ABO compatible with the product. Only 35 patients (14\%) received incompatible plasma due to a group $\mathrm{B}$ or $\mathrm{AB}$ blood type with no adverse events and no discernable increase in morbidity. One interesting finding was that only $2.5 \%$ of trauma patients actually received emergency plasma transfusion. Likewise, Dartmouth found that only a small percentage of trauma patients $(1.2 \%)$ required emergent transfusion with the majority typing as group $\mathrm{A}$ and group $\mathrm{O}(79 \%) .{ }^{8}$ At this time, Dartmouth provides 4 units of group A plasma as part of their initial trauma pack with no reported hemolytic transfusion reactions. Mayo estimates that the policy potentially decreased $\mathrm{AB}$ plasma use by $97 \% .^{5}$

A weakness and strength of the Massachusetts and Mayo data is the small number of patients that ultimately received $\mathrm{ABO}$-incompatible plasma over a combined 8-year period. Overall, only 58 patients in the two studies were group $\mathrm{B}$ or $\mathrm{AB}$ and only 12 patients required massive transfusion. On average, these patients received between 500 and $750 \mathrm{~mL}$ of incompatible plasma or less than $20 \%$ of their expected total blood volume. In the study by Chhibber and colleagues nearly half of patients could now be treated with prothrombinase complex to reverse warfarin, thereby avoiding plasma exposure completely. ${ }^{2,13}$ Although no hemolysis or other acute adverse events were observed, the number of patients is too small to assess the incidence of other adverse outcomes attributed to nonidentical PLT and plasma transfusions. ${ }^{14,15}$ Finally, Mayo and Massachusetts wisely restricted the use of group A plasma to adult patients and their experience cannot be extrapolated to pediatric populations.

Another concern is the demographics of the trauma populations. Mayo and Dartmouth serve predominantly rural white populations and even the Worcester area is $77 \%$ white, with black persons comprising only $7 \%$ of the population. ${ }^{16}$ Racial diversity causes concern for large urban hospitals with an ethnically diverse patient population and a higher percentage of group B patients. It is hoped that more data may soon become available from the recently closed PROPPR study (Pragmatic Randomized Optimal Platelet and Plasma Ratios). ${ }^{17}$ PROPPR is a multi-institutional prospective study involving 11 academic centers across the United States, including two that dispense group A plasma for emergency release. Finally, the US military manual allows the use of group A plasma in emergencies and could be an additional source of information on safety and patient outcomes. ${ }^{18}$
Despite the paucity of published literature, a poll of attendees at a recent $\mathrm{AABB}$ annual meeting showed that $23 \%$ already use non-AB plasma for emergency release and more than $90 \%$ were considering prethawed group $\mathrm{A}$ plasma for massive transfusion patients. ${ }^{4}$ Some centers using group A plasma employed different approaches to further minimize the risk of ABO-incompatible plasma transfusion. The Detroit Medical Center provides a mix of group $\mathrm{AB}$ and $\mathrm{A}$ plasma (two $\mathrm{AB}$, two $\mathrm{A}$ : four group $\mathrm{O}$ RBCs) in their initial MTP, followed by transition to typespecific or group A plasma for subsequent MTP packs as necessary (B. O’Malley, personal communication, 2013). Other centers withhold group A plasma until several units of group O RBCs are transfused. For example, the University of Texas at San Antonio does not provide group A plasma until the patient has received 8 units of group $\mathrm{O}$ RBCs. ${ }^{4}$

Other mitigation strategies include screening group A plasma for low anti-B titers. Dartmouth currently screens group A plasma and reserves units with titers less than 50 (97\% of units) for their MTP patients. ${ }^{8}$ Mayo does not prescreen their units but documented that $90 \%$ of units had a titer less than $64 .{ }^{10}$ These data are consistent with other data that also show relatively low anti-B titers among group A donors, ${ }^{9,11,12}$ although hemolysis due to high-titer anti-B has been reported with aggressive oral probiotic use. ${ }^{19}$ Practically, plasma units need to be identified and readily available to minimize delays in dispensing plasma, effectively limiting this approach to centers that collect allogeneic blood and can test donor samples during manufacturing. Testing of thawed plasma by transfusion services is impractical due to current plasma bag design and time limitations. Furthermore, ABO titers can vary between testing facility, method, and reagents and cannot accurately predict the risk of hemolysis. ${ }^{12,20}$ Testing of apheresis PLTs shows a wide range of testing practices and titer cutoffs between institutions. ${ }^{12}$

It may be several years before the wisdom of group A plasma in ABO-incompatible adult trauma and massive transfusion patients can be fully assessed and confirmed, although the initial literature suggests its safety. Early published studies have been hampered by small numbers and slow accrual of group $\mathrm{B}$ and $\mathrm{AB}$ patients requiring emergency plasma transfusion., ${ }^{5,13}$ It will take significantly larger patient numbers to examine other clinical outcome measures such as the risk of acute lung injury, infection, and mortality. It is possible that group A plasma may result in less morbidity than $\mathrm{AB}$ plasma based on recent studies in trauma patients., ${ }^{5,15}$ Future studies will also need to address whether there are volume thresholds for incompatible plasma transfusion and the impact on subsequent transfusion support. In patients who were massively transfused with group $\mathrm{O}$ whole blood, transfusion of typespecific blood was withheld for a period of 2 weeks due to the risk of hemolysis. ${ }^{21}$ 


\section{CONFLICT OF INTEREST}

The author has disclosed no conflicts of interest.

Laura Cooling, MD, MS

e-mail:lcooling@med.umich.edu

Department of Pathology

University of Michigan

Ann Arbor, MI

\section{REFERENCES}

1. Carson TH. Standards for blood banks and transfusion services. 29th ed. Bethesda (MD): American Association of Blood Banks; 2014.

2. American Association of Blood Banks. Association Bulletin \#14-02. TRALI risk mitigation for plasma and whole blood for allogeneic transfusion. 2014 [cited 2014 May 8]. Available from: http://www.aabb.org/resources/publications/ bulletins/Documents/ab14-02.pdf

3. Eder AF, Dy B, Perez J, et al. The residual risk of transfusion-related acute lung injury risk at the American Red Cross (2008-2011): limitations of a predominantly male-donor plasma mitigation strategy. Transfusion 2013; 53:1442-9.

4. Research in progress: use of potentially incompatible group A thawed plasma in trauma patients. $2013 \mathrm{AABB}$ Annual Meeting, Denver, CO, October 13, 2013.

5. Zelinski MD, Johnson PM, Jenkins D, et al. Emergency use of prethawed group A plasma in trauma patients. J Trauma Acute Care Surg 2013;74:69-75.

6. Inaba K, Branco BC, Rhee P, et al. Impact of plasma transfusion in trauma patients who do not require massive transfusion. J Am Coll Surg 2010;210:957-65.

7. Yazer M, Eder AF, Land KJ. How we manage AB plasma inventory in the blood center and transfusion service. Transfusion 2013;53:1627-33.

8. Mehr CR, Gupta R, von Recklinghausen FM, et al. Balancing risk and benefit: maintenance of a thawed group A plasma inventory for trauma patients requiring massive transfusion. J Trauma Acute Care Surg 2013;74:1425-31.

9. Isaak EJ, Tchorz KM, Lang N, et al. Challenging dogma: group A donors as "universal plasma" donors in massive transfusion protocols. Immunohematology 2011;27:61-5.
10. Tauscher C, Bendix B, Jacob EK, et al. Transfusing A plasma to trauma patients: should we worry about anti-B [abstract]. Transfusion 2013;53S:192A.

11. Cooling LL, Downs TA, Butch SH, et al. Anti-A and anti-B titers in pooled group $\mathrm{O}$ platelets are comparable to apheresis platelets. Transfusion 2008;48:2106-13.

12. Cooling L. ABO and platelet transfusion therapy. Immunohematology 2007;23:20-33.

13. Chhibber V, Green M, Vauthrin M, et al. Is group A plasma suitable as the first option for emergency release transfusion? Transfusion 2014;54:1751-5.

14. Shanwell A, Andersson TM, Rostgaard K, et al. Posttransfusion mortality among recipients of ABO-compatible but non-identical plasma. Vox Sang 2009;96:316-23.

15. Inaba $\mathrm{K}$, Branco $\mathrm{BC}$, Rhee $\mathrm{P}$, et al. Impact of $\mathrm{ABO}$-identical vs ABO-compatible nonidentical plasma transfusion in trauma patients. Arch Surg 2010;145:899-906.

16. Worcester Regional Research Bureau. Report 13-01. Worcester's demographic trends: 2010 census. February 2013 [cited 2014 Mar 28]. Available from: http://www .wrrb.org

17. National Institute of Health. Pragmatic, Randomized optimized platelet and plasma ratios. 2012. [cited 2014 Mar 30]. Available from: http://www.clinicaltrials.gov/ct2/ show/NCT01545232?term=PROPPR\&rank=1

18. Office of The Surgeon General, Borden Institute. Chapter 33, Battlefield transfusions. In: Cubano MA, Lenhart MK, editors. Emergency war surgery. 4th revision. Fort Sam Houston (TX): Office of The Surgeon General, Borden Institute; 2013. p. 467-88 [cited 2014 March 20]. Available from: http://www.cs.amedd.army.mil/borden

19. Daniel-Johnson J, Leitman S, Klein H, et al. Probioticassociated high-titer anti-B in a group A platelet donor as a cause of severe hemolytic transfusion reactions. Transfusion 2009;49:1845-9.

20. Karafin MS, Blagg L, Tobian AA, et al. ABO antibody titers are not predictive of hemolytic reactions due to plasmaincompatible platelet transfusions. Transfusion 2012;52: 2087-93.

21. Berseus O, Boman K, Nessen S, et al. Risks of hemolysis due to anti-A and anti-B caused by the transfusion of blood or blood components containing ABO-incompatible plasma. Transfusion 2013;53:114S-123S. 